\title{
RELIABILITY ASSESSMENT OF A ROBUST DESIGN UNDER UNCERTAINTY FOR A 3-D FLEXIBLE WING
}

\author{
Clyde R. Gumbert* \\ NASA Langley Research Center, Hampton, VA 23681 \\ Gene J.-W. Hou ${ }^{\dagger}$ \\ Old Dominion University, Norfolk, VA 23529-0247 \\ Perry A. Newman ${ }^{\ddagger}$ \\ NASA Langley Research Center, Hampton, VA 23681
}

\begin{abstract}
The paper presents reliability assessment results for the robust designs under uncertainty of a 3-D flexible wing previously reported by the authors. Reliability assessments (additional optimization problems) of the active constraints at the various probabilistic robust design points are obtained and compared with the constraint values or target constraint probabilities specified in the robust design. In addition, reliability-based sensitivity derivatives with respect to design variable mean values are also obtained and shown to agree with finite difference values. These derivatives allow one to perform reliabilitybased design without having to obtain second-order sensitivity derivatives. However, an inner-loop optimization problem must be solved for each active constraint to find the most probable point on that constraint failure surface.
\end{abstract}

\section{Introduction}

In references 1 and 2, domains of uncertainty-based design were classified with respect to the impact and frequency of an event as shown in figure 1 . We quote several paragraphs from reference 1 regarding "robust design" and "reliability-based design":

"The two major classes of uncertainty-based design problems are robust design problems and reliabilitybased design problems. A robust design problem is one in which a design is sought that is relatively insensitive to small changes in the uncertain quantities. A reliability-based design problem is one in which a design is sought that has a probability of failure that is less than some acceptable (invariably small) value. The same abstract mathematical formulation can be used to describe both robust design and reliabilitybased design. However, their domains of applicability are rather different.

"Figure 1 illustrates these domains. The two major factors are the frequency of the event and the impact of the event. No system is viable if everyday fluctuations can lead to catastrophe. Instead, one would like the

\footnotetext{
*Research Engineer, Multidisciplinary Optimization Branch M/S 159, clyde.r.gumbert@nasa.gov

${ }^{\dagger}$ Professor, Department of Mechanical Engineering, AIAA member, ghou@odu.edu

† Senior Research Scientist, Multidisciplinary Optimization Branch, M/S 159, perry.a.newman@nasa.gov
}

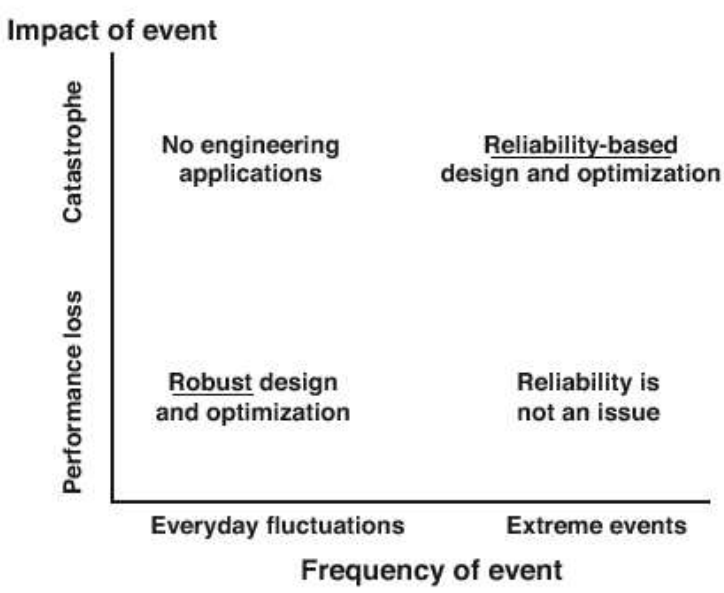

Fig. 1 Uncertainty-based design domains (courtesy of Luc Huyse, taken from Ref. 1).

system to be designed such that the performance is insensitive, i.e., robust, to everyday fluctuations. On the other hand, one would like to ensure that the events that lead to catastrophe are extremely unlikely. This is the domain of reliability-based design. In both cases, the design risk is a combination of the likelihood of an undesired event and the consequences of that event. An example of risk in the robust design context is the likelihood that the aircraft design will fail to meet the aerodynamic performance targets and [that the manufacturer] will consequently lose sales and perhaps even 
go bankrupt. An example of risk in the reliabilitybased design context is the probability that a critical structural component will fail, which could lead to the loss of the vehicle or spacecraft, payload, and passengers, and to potential lawsuits.

"As figure 2 illustrates, robust design is concerned with the event distribution near the mean of the probability density function, whereas reliability-based design is concerned with the event distribution in the tails of the probability density function. Obviously, it is much more difficult to accurately characterize the tail of a distribution than the center of the distribution. An additional consideration in distinguishing between robustness and reliability is that the mathematical techniques used for solving robust design problems are considerably different from those used for solving reliability-based design problems. The mathematical methods for robust design procedures are less well developed than those for reliability-based design procedures, and this work is still largely confined to academic studies."

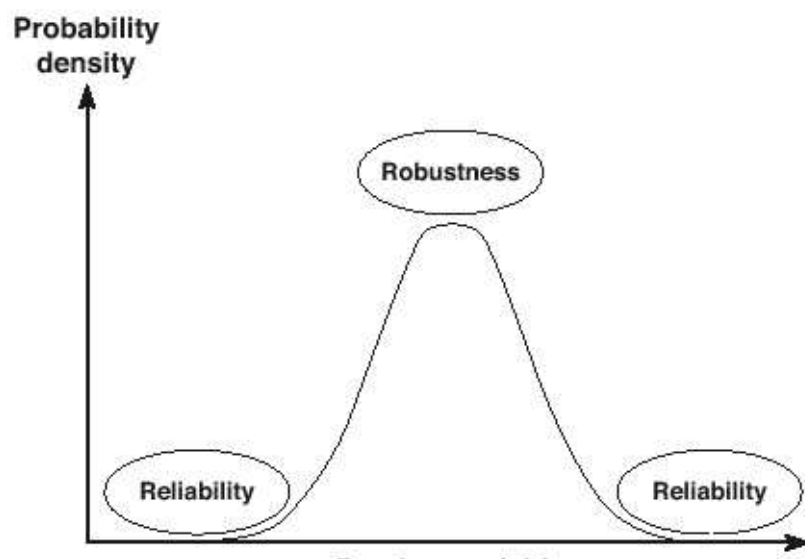

Random variable

Fig. 2 Reliability versus robustness in terms of the probability density function (taken from Ref. 1).

The recent interest in multidisciplinary analysis and design for aerospace vehicles has indicated the need for using uncertainty-based methods ${ }^{1}$ in all design phases from low to high fidelity. Three recent AIAA papers ${ }^{3-5}$ are pertinent to the preceding discussion of reliability versus robustness in regard to aerospace vehicle design under uncertainty using high-fidelity analyses. Reference 3 presents gradient-based robust design optimization results for a $3-\mathrm{D}$ flexible wing based on Euler CFD and FEM structural simulations and using the first-order second moment (FOSM) statistical approximation. Reference 4 presents gradient-based, reliability-based design optimization results for a 3D flexible wing based on Euler computational fluid dynamics (CFD) and finite element method (FEM) structural simulations and using the first-order reliability method (FORM). Interested readers are referred to references 3 and 4 and the sources cited therein for more background on design under uncertainty.

Reference 5 discusses three different probabilistic approaches with respect to accuracy, stability and efficiency. The basic difference in these probabilistic optimization approaches is in the details of how the probabilistic constraints are satisfied. The robust design approach, such as the Approximate Moment Approach (AMA) in reference 5, evaluates the constraint sensitivity derivatives at the mean value. The reliability-based design approaches, such as the Reliability Index Approach (RIA) in reference 5 and the Performance Measure Approach (PMA) in reference 5 evaluate the constraint sensitivities at the most probable point (MPP) on the (constraint) failure surface. Observations and results for AMA and PMA are given in reference 5 for an algebraic example and a vehicle side impact crash-worthiness example.

References 4 and 5 both make the case for favoring the use of reliability-based design optimization approach. This choice also appears to be the preferred practice in the various structural disciplines. ${ }^{6}$ Perhaps whenever real (physical) structures are involved in Multidisciplinary Optimization (MDO), one must resort to using reliability-based methods. However, we agree with reference 1 that there is an uncertaintybased design domain where robust design optimization is valid and useful.

We present several reliability assessments of the $3-\mathrm{D}$ flexible wing robust design results given in reference 3 . We use conventional RIA, PMA and a new PMA/RIA to evaluate the active constraints at the various probabilistic robust design points; we compare these results with the constraint values or the target constraint probabilities specified in the robust design. In addition, we obtain reliability-based sensitivity derivatives with respect to the design variable mean values and show that they agree with finite difference values. These derivatives eliminate the need for second-order sensitivity derivatives to perform reliability-based design. However, inner-loop optimization problems must be solved to find the MPP for each active constraint.

\section{Uncertainty Methods}

Only a brief outline of the methods used herein is given. Details of our robust design method can be found in reference 3 and the references cited therein. Details of our reliability assessment methods can be found in reference 7 . All of the methods considerred here are implemented using gradient-based optimization techniques.

\section{Robust Design (FOSM)}

The first step in the FOSM analyses is to approximate the system output solutions of interest in Taylor series form. These approximations are formed to estimate the output value for small deviations of the input. Given input random variables $b=\left\{b_{1}, \cdots, b_{n}\right\}$ 
with means $\bar{b}=\left\{\bar{b}_{1}, \cdots, \bar{b}_{n}\right\}$ and standard deviations $\sigma=\left\{\sigma_{1}, \cdots, \sigma_{n}\right\}$, and system output function $\mathrm{F}$, the first-order Taylor series approximations are

$$
F(b)=F(\bar{b})+\sum_{i=1}^{n} \frac{\partial F}{\partial b_{i}}\left(b_{i}-\bar{b}_{i}\right)
$$

In an approximate moment approach (AMA), one obtains expected values for the mean (first moment) and variance (second moment) of the output function, $\mathrm{F}$; these values depend on the sensitivity derivatives and input variances $\sigma_{i}{ }^{2}$. The mean of the output function $\bar{F}$ and variance $\sigma_{F}^{2}$ are approximated as

$$
\begin{aligned}
\bar{F} & =F(\bar{b}) \\
\sigma_{F}^{2} & =\sum_{i=1}^{n}\left(\frac{\partial F}{\partial b_{i}} \sigma_{i}\right)^{2}
\end{aligned}
$$

where the sensitivity derivatives, $\partial F / \partial b_{i}$, are evaluated at the mean values $\bar{b}$. These sensitivity derivatives are obtained using the automatic differentiation tool ADIFOR. ${ }^{8,9}$

For robust design, design variables are the mean values $\bar{b}=\left\{\bar{b}_{1}, \cdots \bar{b}_{n}\right\}$ with $\bar{b}$ assumed statistically independent and normally distributed with standard deviations $\sigma_{i}$. The CFD state and structural equilibrium equation residuals $R$ are deemed to be satisfied at the mean values of the state variables $\bar{Q}$ and design variables $\bar{b}$ such that $R(\bar{Q}, \bar{b})=0$. The objective function $\Psi$ is cast in terms of expected values and becomes a function of $\bar{F}$ and $\sigma_{F}$. The system constraints are cast into a probabilistic statement: the probability that the constraints are satisfied is greater than or equal to a desired or specified target probability; that is, $P(g \leq 0)>P_{t}$. This probability statement is transformed to a constraint involving mean values and standard deviations under the assumption that the variables involved are normally distributed. The robust optimization can be expressed as

$$
\begin{aligned}
\min & \Psi=\Psi\left(\bar{F}, \sigma_{F} ; \bar{Q}, \bar{b}\right) \\
\text { subject to } & g(\bar{F} ; \bar{Q}, \bar{b})+k \sigma_{g} \leq 0
\end{aligned}
$$

where $k$ is the number of standard deviations $\sigma_{g}$ that the constraint $g$ must be displaced to achieve the desired or specified target probability $P_{t}$. For the FOSM approximation, $\sigma_{F}$ and $\sigma_{g}$ require first-order sensitivity derivatives following the form given in Eqs. (2).

\section{Reliability Assessment (FORM)}

The constraint functions $g$ in Eq. (3) are considered as the limit-state equations in reliability analysis. If they are relatively smooth, then it is expected that FORM may provide adequate accuracy for reliability assessment. In FORM, reliability analysis requires a transformation from the original random design variable $b_{i}$ to the standard normal random variable $u_{i}$,

$$
u_{i}=\frac{b_{i}-\overline{b_{i}}}{\sigma_{i}}
$$

where $\bar{b}_{i}$ and $\sigma_{i}$ are the mean and standard deviation of $b_{i}$. Thus, the limit-state equations $g(b, Q)=0$ can be reformulated as $g(u, Q(u))=0$, or in abbreviation, $g(u)=0$. Three variations of FORM are used here for reliability assessment.

\section{Reliability Index Approach (RIA)}

An efficient FORM method, the Hasofer-LindRackwitz-Fiessler (HL-RF) method ${ }^{10,11}$ is used here to analyze the failure probabilities of constraints at the optimal designs of Eq. (3) for the various $k$ values. The reliability index $\beta^{*}$ is then sought to be the shortest distance from the origin to the surface of $g(u)=0$. This point on the surface is called the Most Probable Point (MPP) of failure. Mathematically, this search is expressed as a minimization problem,

$$
\begin{aligned}
& \min _{u} \quad \beta=\sqrt{u^{T} u} \\
& \text { subject to }
\end{aligned}
$$

$$
g(u)=0
$$

which is the basis of the RIA. ${ }^{5}$

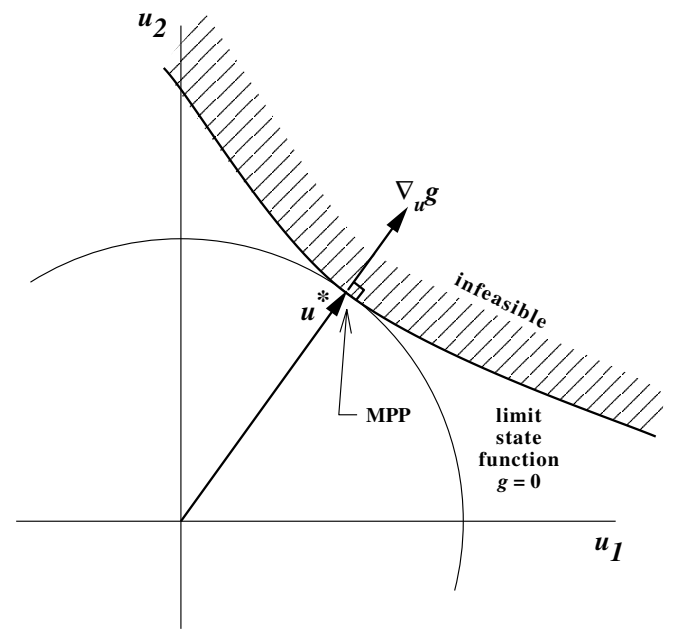

Fig. 3 Most Probable Point (MPP) in standard normal space for two variables.

The HL-RF method used here is based on the observation that the final solution $u^{*}$ is in the same direction as the gradient of a limit-state equation $\nabla_{u} g(u)$, as depicted in Fig. 3 for two random variables. This observation leads to a recursive equation,

$$
u^{i+1}=\beta^{i+1} n^{i}
$$

where $n^{i}$ is the unit direction of $\nabla_{u} g\left(u^{i}\right)$ and $\beta^{i+1}$ is the updated reliability index which is obtained as

$$
\beta^{i+1}=\beta^{i}+\Delta \beta
$$


and the $\Delta \beta$ is obtained as the first-order correction of a nonzero $g\left(u^{i}\right)$,

$$
\Delta \beta=-\frac{g\left(u^{i}\right)}{\left(\nabla_{u} g\right)^{T} n^{i}}
$$

where the term, $\nabla_{u} g$, is evaluated at $u^{i}$. The convergence of the HL-RF method is achieved when the root-mean-square of $\left(u^{i+1}-u^{i}\right)$ and the absolute values of $g\left(u^{i+1}\right)$ and $\Delta \beta$ are less than some prescribed tolerances.

The convergence of Eqs. (6) and (7) gives the reliability index $\beta^{*}$ and the most probable failure point $u^{*}$ which can be used to compute the failure probability of the limit-state equation of concern as

$$
P_{f}=P(g>0)=\Phi\left(-\beta^{*}\right)
$$

where $\Phi$ is the standard normal cumulative density function.

The derivative of the probability of failure can be calculated by differentiating the above equation with respect to the mean value of any design variable as

$$
\frac{d P_{f}}{d \bar{b}_{i}}=p\left(-\beta^{*}\right) \frac{d \beta^{*}}{d \bar{b}_{i}}
$$

where $p\left(\beta^{*}\right)$ is the Probability Density Function (PDF) at $\beta^{*}$ and the derivative, $\frac{d \beta^{*}}{d b_{i}}$, is derived based on the sensitivity of the optimum solution ${ }^{7,12,13}$ as

$$
\frac{d \beta^{*}}{d \bar{b}_{i}}=-\left(\frac{\beta^{*}}{\left(\nabla_{u} g\right)^{T} u^{*}}\right) \frac{\partial g}{\partial \bar{b}_{i}}
$$

The derivatives of the probability of failure with respect to the standard deviations can be obtained in a similar way. Note that in Eq. (11), $\nabla_{u} g$ and $\frac{\partial g}{\partial \bar{b}_{i}}$ are evaluated at the MPP.

It has been reported in the literature that the RIA fails to converge for some problems. ${ }^{14}$ A PMA method $^{14}$ and an alternative RIA method that can overcome such a deficiency are studied in the following subsections.

\section{Performance Measure Approach (PMA)}

In PMA, the objective is to compute the first-order probabilistic performance measure, $g_{p}^{*}$. It is defined as the offset of the performance, $g(b, Q)$, so that the shortest distance between the limit-state equation $g(b, Q)-g_{p}^{*}=0$ and the origin of the $\mathrm{u}$-space is equal to a given target reliability, $\Phi\left(\beta_{0}\right)$. This offset $g_{p}^{*}$ can also be found as the smallest value of $g$ that is tangent to the target reliability surface represented by a sphere constraint, $\|u\|=\beta_{0}=\sqrt{u^{T} u}$. Mathematically, the first-order probabilistic performance measure is obtained as the objective of an optimization problem:

$$
\begin{array}{cl}
\underset{u}{\min _{u}} & g(u) \\
\text { subject to } & \\
& \sqrt{u^{T} u}-\beta_{0}=0
\end{array}
$$

where the constraint requires the solution to achieve the targeted reliability index. This optimization can be viewed as inverse to that of Eq. (5).

The PMA method is also based on the observation that the final solution $u^{*}$ is in the same direction as the gradient of the limit-state equation $\nabla_{u} g(u)$ as depicted in Fig. 3 for two random variables. However, in PMA, because the length of vector $u^{*}$ is limited to be the given $\beta_{0}$ as prescribed in the constraint, a very simple recursive formula can then be devised to find the $u^{*}$ :

$$
u^{i+1}=\beta_{0} n^{i}
$$

This algorithm is quite efficient. However, Choi and Youn ${ }^{14}$ experienced convergence difficulty of Eq. (13) when the limit-state equation exhibits concavity. They then replace the current unit direction $n^{i}$ in Eq. (13) by the average of the last three consecutive ones. ${ }^{14} \mathrm{We}$ adopt the same procedure in our PMA.

In PMA, derivatives of the performance measure $g_{p}^{*}$ are obtained from derivatives of the optimum solution $^{7,12,13}$ as

$$
\frac{d g_{p}}{d \overline{b_{i}}}=\frac{\partial g}{\partial \bar{b}_{i}}
$$

and

$$
\frac{d g_{p}^{*}}{d \beta_{0}}=\frac{\left(\nabla_{u} g\right)^{T} u^{*}}{\beta_{0}}
$$

where $\nabla_{u} g$ and $\frac{\partial g}{\partial b_{i}}$ are evaluated at the MPP.

\section{Alternate Algorithm for RIA (PRIA)}

The key motivation of this new algorithm is the observation that the target $\beta_{0}$ of PMA is identical to the reliability index $\beta^{*}$ of RIA if the performance measure $g_{p}^{*}$ in PMA reaches zero value. To achieve a zero $g_{p}^{*}$, the new algorithm uses Eq. (15) to estimate the amount of the change in $\beta_{0}$ needed to reduce the nonzero value of $g^{*}$;

$$
\Delta g=\frac{\left(\nabla_{u} g\right)^{T} u}{\beta_{0}} \Delta \beta
$$

Let $\Delta g^{*}=0-g_{p}^{*}$ be the gap between zero and the current value of $g_{p}^{*}$. The change in target $\beta_{0}$ that is required to achieve a zero $g_{p}^{*}$ is then estimated by Eq. (16) as

$$
\Delta \beta=-g_{p}^{*}\left(\frac{\beta_{0}}{\left(\nabla_{u} g\right)^{T} u}\right)
$$

The updated reliability index $\beta+\Delta \beta$ will yield a new $g_{p}^{*}$ that is closer to zero, at least in the first order sense. Repeated use of Eq. (17) can guide the PMA search to arrive at $g_{p}^{*}=0$. In short, the new algorithm is derived based on the PMA reported in the previous subsection. Thus, this method can handle the concavity of the limit-state equation as the underlying PMA can. The new algorithm, called PMA-based RIA (PRIA) can be summarized as follows:

Step 1: Start with an initial target $\beta^{i}=\beta_{0}$ and initial values of $u^{i}=u_{0}$. 
Step 2: Follow the PMA procedure ${ }^{7}$ to obtain the converged performance measure $g_{p}^{* i}$.

Step 3: Compute $\Delta \beta^{i}$ by Eq. (17) which is evaluated at $u^{i}$ and $\beta^{i}$.

Step 4: Update $\beta^{i+1}=\beta^{i}+\Delta \beta^{i}$.

Step 5: Repeat steps 2-4 until $\Delta \beta$ and $g_{p}^{*}$ achieve the tolerances required for convergence.

The above algorithm is similar to the one presented by Du and Chen. ${ }^{15}$ Both trace the MPP locus to locate the $\beta^{*}$. Nevertheless, the present method follows the tangential direction of the MPP locus, whereas that of reference 15 follows an extrapolated MPP locus. Note that Eq. (17) is in the same form as Eq. (8); however, Eq. (17) is only valid at the optimum of the PMA. Thus, it represents a slope on the MPP locus, whereas Eq. (8) does not.

\section{3-D Flexible Wing Problems}

The topic of this paper is the application of the three reliability assessment methods to results of robust design for a 3-D flexible wing. Reliability assessments are carried out for constraints that are near the limit of feasibility. In this section, we describe the models and computational tools used for the robust optimizations and reliability assessments. Then we present the robust design cases to which the reliability assessments are to be applied.

\section{Robust Design (FOSM)}

The initial design point for the robust optimizations were results of previous deterministic design optimizations. The trapezoidal-planform, semispan wing and its associated shape parameters are shown in Fig. 4; more details can be found in reference 3 . The relative sizes of the skin thickness, the web thicknesses, and the truss cross section areas are fixed within each structural zone depicted in Fig. 4. A scaling factor $\Gamma_{n}$ is assigned to change the thickness and area of all structural elements in zone $n$.

In the two-design-variable (2DV) examples, the wing sections are held fixed and vary linearly from an NACA 0012 at the root to an NACA 0008 at the tip, which is then rounded. The size of the structural elements is also held fixed in the 2DV examples. The two planform design variables are the tip setback $x_{t}$ and the tip chord $c_{t}$. Although these two planform variables are not typically subject to significant variance, they are ascribed some uncertainty here to illustrate the techniques. For the $2 \mathrm{DV}$ cases shown here, a coefficient of variation $\frac{\sigma_{i}}{\bar{b}_{i}}=0.01$ was arbitrarily chosen for both DVs.

In the four-design-variable (4DV) examples, the uncertain design variables were determined by examining both sensitivity derivatives and reasonable expected variances of the available parameters. As can be seen from Eq. (2), the size of an output function variance depends on the size of the input parameter variances weighted by the square of the output function sensitivity derivatives with respect to those input parameters. For reasonable input coefficients of variation of $0.1 \%$, the larger sensitivity derivatives with respect to root airfoil thickness $t_{r}$ and camber $z_{r}$ appeared to provide a more realistic representation of practical uncertainty than the sensitivity derivative with respect to other shape parameters. Similarly, the sensitivity derivatives for the two inboard structural sizing parameters are larger than the others. The four parameters chosen as uncertain design variables were the root airfoil section maximum thickness $t_{r}$, the root airfoil section maximum camber $z_{r}$, and the structural sizing factors for the two inboard regions, $\Gamma_{1}$ and $\Gamma_{2}$, as shown in Fig. 4. For the 4DV cases herein, a coefficient of variation, $\frac{\sigma_{i}}{b_{i}}=0.001$, was chosen for all four design variables.

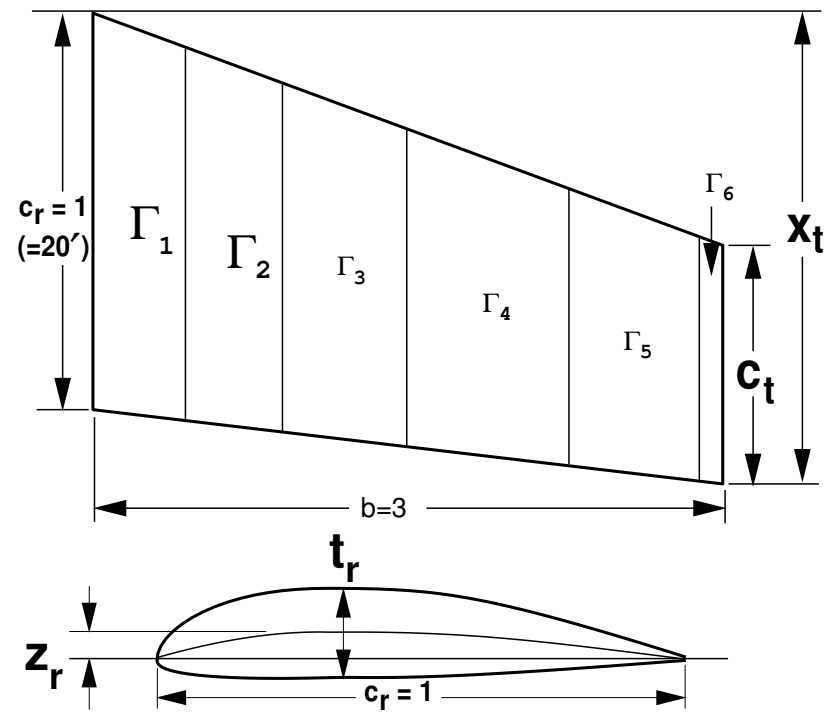

Fig. 4 Wing geometry and sizing parameterization.

The objective function to be minimized is the negative of the square of the lift-to-drag ratio, $-(L / D)^{2}$, for $M_{\infty}=0.8, \alpha=1^{\circ}$, representative of cruise conditions for a transport aircraft. Coupled solution-dependent and geometric constraints were imposed. The solutiondependent constraints were

- lower limit on the difference between the total lift and the structural weight, $L-W$, where $L=$ $C_{L} * S * q_{\infty}$

- upper limit on compliance, the work done by the aerodynamic loads to deflect the structure, $V=$ $\oint p u \cdot \hat{n} d s$

- upper limit on pitching moment, $C_{m}$, in lieu of a trim constraint

The purely geometric constraints were 
- minimum leading edge radius, in lieu of a manufacturing requirement

- side constraints (bounds) on the active design variables

State variables for aerodynamics and structures were determined from the fluid-flow conservation laws (Euler equations) and the structural equilibrium conditions (for FEM), respectively.

In the present examples, the objective function is taken as $-(L / D)^{2}$ and the FOSM approximation to the variance appears in the robust version. Thus when the FOSM approximation of the standard deviation is used in the constraint, the robust optimization problem is given as

$$
\min \Psi=-(\bar{L} / \bar{D})^{2}-\sum_{i=1}^{n}\left(\frac{\partial(L / D)}{\partial b_{i}} \sigma_{i}\right)^{2}
$$

subject to

$$
g=\bar{g}+k \sqrt{\sum_{i=1}^{n}\left(\frac{\partial g}{\partial b_{i}} \sigma_{i}\right)^{2}} \leq 0
$$

Note that the robust optimization problem reduces to the conventional or deterministic problem when the standard deviations of the inputs $\sigma_{i}$ are zero.

\section{Computational Tools}

A collection of existing codes was used to perform the computations in this reliability assessment procedure. These codes and data transfers are executed by a separate driver code and scripts. Each code runs independently (some simultaneously on separate processors) and the required data transfers between them are accomplished via data files. The aerodynamic flow analysis code used for this study is a version of the CFL3D code $^{16}$ used in the Euler mode. The gradient version of this code, which was used for aerodynamic sensitivity analysis, was generated by an unconventional application ${ }^{17}$ of the automatic differentiation code $\mathrm{ADIFOR}^{8,9}$ to produce a relatively efficient, direct mode, gradient analysis code. ${ }^{18}$ The surface geometry was generated based on a code that uses the Rapid Aircraft Parameterization Input Design (RAPID) technique developed by Smith et al. ${ }^{19}$ This code was also preprocessed with ADIFOR to generate a code capable of producing sensitivity derivatives as well. A version of the $\mathrm{CSCMDO}^{20}$ code was used to generate the CFD volume mesh needed by the flow analysis code. The associated grid sensitivity derivatives needed by the flow sensitivity analysis were generated with an automatically differentiated version of CSCMDO. ${ }^{21}$ The 45,000 grid point baseline volume mesh required by CSCMDO and used in the present flexible wing examples was obtained with the Gridgen code. The wing surface portion of the mesh is shown

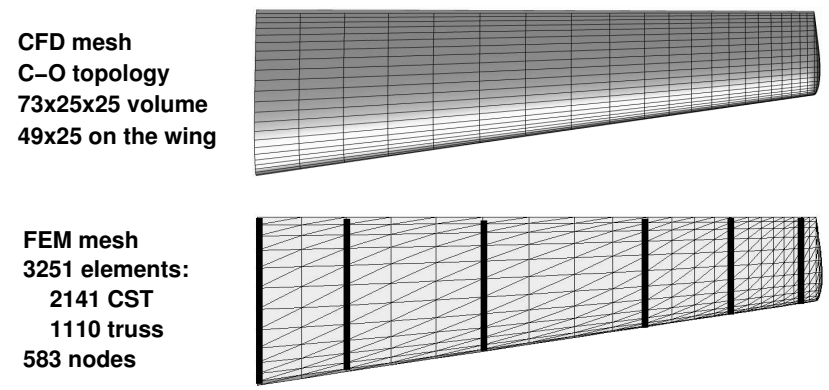

Fig. 5 CFD and FEM computational meshes.

in Fig. 5. This mesh is admittedly quite coarse by current CFD analysis standards. The structural analysis code $^{22}$ used to compute the deflection of the elastic wing was a generic finite element code. The flexible structure for the wing shown in Fig. 5 was discretized by 583 nodes; there were 2,141 constant-strain triangle (CST) elements and 1,110 truss elements. Zone boundaries for the design variables controlling element size are also shown in Fig. 5. Because the elastic deformation was assumed to be small, linear elasticity was deemed to be appropriate. The structural sensitivity equations were derived based on the direct differentiation method. The sensitivity of the aerodynamic forces appears as a term on the right-hand side (RHS) of the deflection sensitivity equations. The derivative of the stiffness matrix in these sensitivity equations was also generated $^{23}$ by using the ADIFOR $^{8,9}$ technique. The coefficient matrix of the structural sensitivity equations was identical to that of the structural equations. Consequently, these structural sensitivity equations were solved efficiently by backward substitution with a different term on the RHS for each sensitivity. At the wing surface, i.e., the interface where aerodynamic load and structural deflection information is transferred, surface nodes of the FEM structural model were assumed to be a subset of the CFD aerodynamic surface mesh points for this application (see Fig. 5). This lack of generality allowed for simplifications in the data transfers; although it is an important issue, it was not deemed crucial for these robust optimization and reliability assessment demonstrations.

\section{DV Robust Results}

Table 1 and Fig. 6 present the results for the 2DV deterministic optimization and robust optimizations for several values of $k$. An increase of the parameter $k$ represents an increase in the specified target probability and therefore the probability that the constraints are met. Assuming a normal Gaussian distribution of the output variables, values of $k=1,2,3$, and 4 would represent target probabilities of $P_{t}=84.13$, $97.73,99.87$, and $99.998 \%$, respectively. The mean values of the constraint functions $\bar{g}$ are shown along with the robust constraint values $g$ in Table 1 . The mean values of the constraints are shown as bars in Fig. 6. The circles represent the contribution of the 
Table 1 2DV robust design problem results.

\begin{tabular}{|c|c|c|c|c|c|c|c|c|c|}
\hline & $\begin{array}{l}\text { Deter- } \\
\text { ministic } \\
\text { solution }\end{array}$ & \multicolumn{2}{|c|}{$\begin{array}{c}\text { Robust } \\
\text { solution, } \\
\quad k=1\end{array}$} & \multicolumn{2}{|c|}{$\begin{array}{c}\text { Robust } \\
\text { solution, } \\
\quad k=2\end{array}$} & \multicolumn{2}{|c|}{$\begin{array}{c}\text { Robust } \\
\text { solution, } \\
k=3\end{array}$} & \multicolumn{2}{|c|}{$\begin{array}{c}\text { Robust } \\
\text { solution, } \\
k=4\end{array}$} \\
\hline \multicolumn{10}{|c|}{ Design variable inputs } \\
\hline$c_{t}$ & 1.130 & \multirow{2}{*}{\multicolumn{2}{|c|}{$\begin{array}{l}1.131 \\
1.917\end{array}$}} & \multicolumn{2}{|c|}{1.158} & \multicolumn{2}{|c|}{1.117} & \multicolumn{2}{|c|}{1.060} \\
\hline$x_{t}$ & 1.940 & & & \multicolumn{2}{|c|}{1.932} & \multicolumn{2}{|c|}{1.838} & \multicolumn{2}{|c|}{1.686} \\
\hline \multicolumn{10}{|l|}{ Responses } \\
\hline obj & -10.0 & \multicolumn{2}{|c|}{-9.94} & \multicolumn{2}{|c|}{-9.90} & \multicolumn{2}{|c|}{-9.82} & \multicolumn{2}{|c|}{-9.56} \\
\hline & & $\bar{g}+k \sigma_{g}$ & $\bar{g}$ & $\bar{g}+k \sigma_{g}$ & $\bar{g}$ & $\bar{g}+k \sigma_{g}$ & $\bar{g}$ & $\bar{g}+k \sigma_{g}$ & $\bar{g}$ \\
\hline$g(L-W)$ & -0.0007 & 0.000127 & -0.0176 & 0.000032 & -0.0357 & -0.000225 & -0.0503 & -0.00607 & -0.0669 \\
\hline$g\left(C_{m}\right)$ & -0.920 & -0.915 & -0.921 & -0.924 & -0.936 & -0.895 & -0.914 & -0.858 & -0.886 \\
\hline$g(V)$ & -0.0966 & -0.0630 & -0.0802 & -0.0257 & -0.0603 & 0.00569 & -0.0525 & 0.00133 & -0.0535 \\
\hline
\end{tabular}

uncertainty to the robust constraint functions. Active constraint values are those for which the circles lie near end of the bars. That is, in Fig. $6, g(L-W)$ is active for all values of $k$ whereas $g(V)$ is active only for $k=3$ and 4 . Mean values of the constraint function that are greater (less negative) than the values at the circles would indicate violated robust constraints; that is, the probability of those constraints being satisfied would be less than the target probability. The changes due to seemingly small uncertainty produce substantial changes in the constraints, which must be accounted for in the optimization.

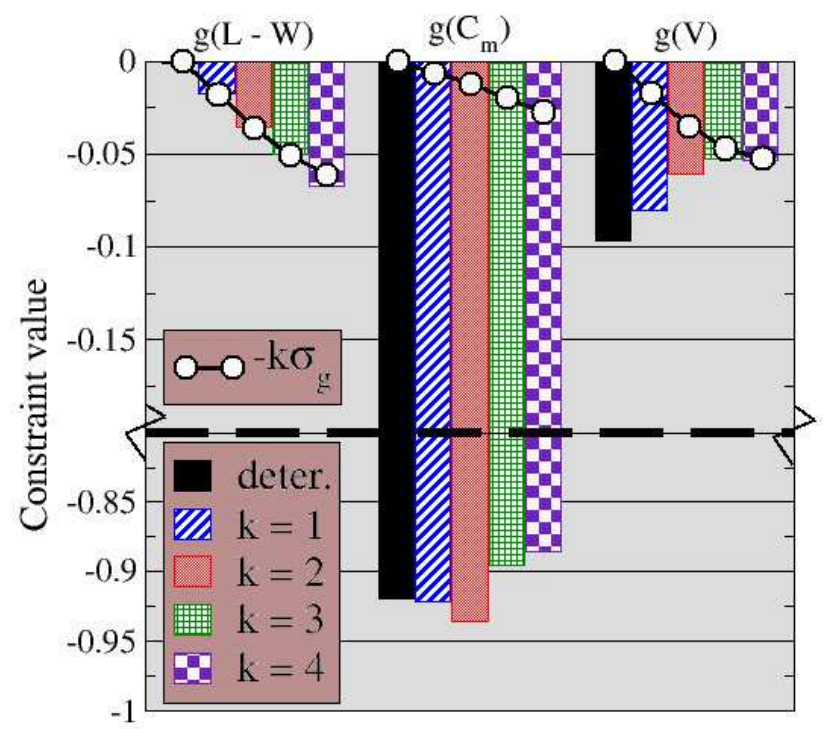

Fig. $62 \mathrm{DV}$ robust optimization results: mean value of constraints.

\section{DV Robust Results}

Table 2 and Fig. 7 present the results for the $4 \mathrm{DV}$ deterministic optimization and robust optimizations ${ }^{3}$ in the same manner as that described above for the $2 \mathrm{DV}$ cases. For all of the $4 \mathrm{DV}$ cases, $k=1,2$, and 3 , the payload and pitching moment constraints are active, but the compliance constraint is not. For the $k=2$ case, although the pitching moment constraint is active, it is not tight. As in the 2DV cases, the changes due to seemingly small uncertainty produce substantial changes in the constraints, which must be accounted for in the optimization. The mean values of the constraints are shown in Fig. 7; and, as in Fig. 6, the circles represent the contribution of the uncertainty to the robust constraint functions.

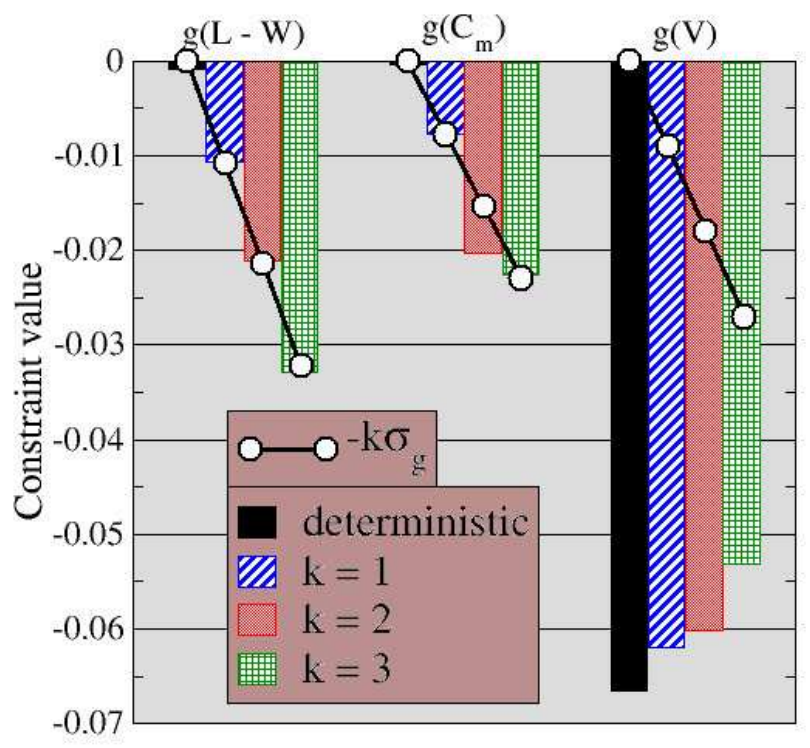

Fig. 7 4DV robust optimization results: mean value of constraints.

\section{Sample Results \& Discussion}

\section{Reliability Assessment of Robust Design (FORM)}

Reliability assessments of the robust design results for the 3-D flexible wing described previously were performed using three previously discussed approaches based on FORM: the Reliability Index Approach (RIA), the Performance Measure Approach (PMA), and the PMA-based RIA (PRIA). These reliability assessments were performed for the constraints that were active in the robust designs. Results corresponding to $k=1,2,3$, and 4 for the $2 \mathrm{DV}$ examples are shown in Table 3. Similarly, results corresponding to 
Table $24 \mathrm{DV}$ robust design problem results.

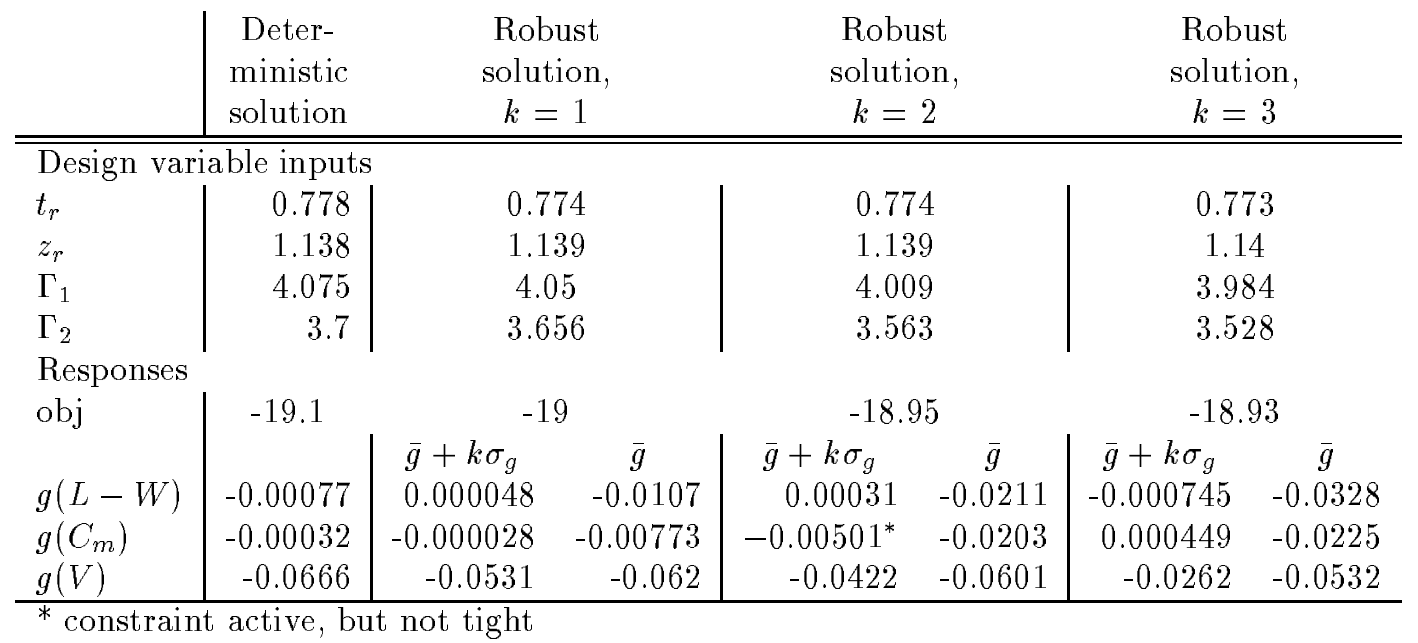

$k=1,2,3$ for the $4 \mathrm{DV}$ examples are shown in Table 4 . In Tables 3 and 4 , the first column indicates the constraint for which the reliability assessments were performed. The second column shows the PMA result $g_{p}^{*}$ at the robust design point. The third column is the final robust constraint value obtained from the robust design process. The PMA assessment of the robust design compares constraint values in these two columns. The fourth and fifth columns are the value of $k$ and its corresponding target probability $P_{t}$ in the robust design. The remaining columns are for RIA method results. The sixth and seventh columns, under the heading "HL-RF", show the reliability index $\beta$ and its associated probability of feasibility $\left(1-P_{f}\right)$ from the HL-RF method at the robust design point. The last two columns, under the heading "PRIA", show the same results using the PMA-based RIA method. The RIA assessments of the robust design compare $\beta$ with $k$ and the probability of feasibility with robust design target probabilities. At absolute convergence the two RIA methods should provide identical results; however, the forms of the convergence criteria are different and the tolerances are finite for the results presented. In the following two subsections we will discuss the results shown in the tables for the 2DV and $4 \mathrm{DV}$ cases.

\section{Two-design-variable case results}

For the robust designs, the payload constraint is active in all the 2DV cases and the compliance constraint becomes active for the $k=3$ and $k=4$ cases as shown in Fig. 6. The reliability index $\beta$ is obtained for both constraints at each value of $k$ using both RIA methods. Therefore, as previously indicated, an optimization problem must be solved to find the MPP for each active constraint. The reliability index $\beta$ is thus obtained for both constraints at the design point resulting from the robust design process for each $k$ and compared with the robust design target values of probability $P_{t}$ as given by $k$. These results are shown in
Table 3 for the 2DV examples. The results indicate that the robust design apparently deviates from the desired probability as $k$ increases; that is, as the desired reliability increases, the RIA consistently shows a higher obtained probability. The PMA assessment performed at the design point that resulted from the robust design process gives a value of $g_{p}^{*}$ for a target reliability index corresponding to the $k$ used in the robust design. The result can be compared with the final robust constraint values for the robust design.

The PMA results are qualitatively consistent with the PRIA results in that when the PRIA indicates that the robust design results have a reliability greater than that corresponding to the input $k$, the PMA gives a negative limit-state value. Similar consistency is also seen for the PMA and HL-RF RIA results except for the $k=1$ case. Hovever, for this case, the RIA $\beta$ values are very similar and close to $k=1$. Table 3 shows that the RIA values $\beta$ for each case are very similar, but not equal - probably due to finite convergence tolerances.

\section{Four-design-variable case results}

Fig. 7 shows that both the payload constraint $g(L-$ $W$ ) and the pitching moment constraint $g\left(C_{m}\right)$ are active for all $4 \mathrm{DV}$ robust design cases. However, note that the pitching moment constraint $g\left(C_{m}\right)$ is not tight for $k=2$ in the $4 \mathrm{DV}$ example. Therefore, the reliability index $\beta$ from the RIA and PRIA reliability assessments for $g\left(C_{m}\right)$ at that design point is not expected to match the input $k$ for $g\left(C_{m}\right)$ but to be somewhat larger. As with the $2 \mathrm{DV}$ results, note that the PMA and RIA reliability assessment results are qualitatively consistent in that when the RIA indicates that the robust design results have a reliability greater than the reliability associated with the input $k$, the PMA gives a negative limit-state value. Conversely, the limit-state value from PMA is positive when the reliability index from PRIA is less than the input $k$. 
Table 3 Reliability assessment of $2 \mathrm{DV}$ robust design results.

\begin{tabular}{l||r||rcc||cr|rr}
\multicolumn{1}{l}{} & \multicolumn{1}{c}{ PMA } & \multicolumn{3}{c}{ Robust Design } & \multicolumn{3}{c}{ RL-RF } \\
Constraint & \multicolumn{1}{c||}{$g_{p}^{*}$} & $\bar{g}+k \sigma_{g}$ & $k$ & \multicolumn{1}{c|}{$P_{t}$} & $\beta$ & $1-P_{f}$ & $\beta$ & $1-P_{f}$ \\
\hline$g(L-W)$ & 0.000358 & 0.000127 & 1 & .8413 & 1.021 & .8465 & 0.987 & .8383 \\
$g(L-W)$ & -0.000200 & 0.000032 & 2 & .9773 & 2.011 & .9779 & 2.001 & .9773 \\
$g(L-W)$ & -0.00350 & 0.000225 & 3 & .9987 & 3.578 & .9998 & 3.244 & .9994 \\
$g(L-W)$ & -0.0146 & -0.00607 & 4 & .9999 & 5.048 & 1. & 5.058 & 1. \\
$g(V)$ & -0.00793 & -0.00569 & 3 & .9987 & 3.215 & .9993 & 3.578 & .9998 \\
$g(V)$ & -0.00733 & -0.00133 & 4 & .9999 & 4.667 & 1. & 4.664 & 1.
\end{tabular}

Table 4 Reliability assessment of $4 \mathrm{DV}$ robust design results.

\begin{tabular}{|c|c|c|c|c|c|c|c|c|}
\hline \multirow[b]{3}{*}{ Constraint } & \multirow{3}{*}{$\begin{array}{c}\text { PMA } \\
g_{p}^{*}\end{array}$} & \multirow{2}{*}{\multicolumn{3}{|c|}{ Robust Design }} & \multicolumn{4}{|c|}{ RIA } \\
\hline & & & & & & $\mathrm{RF}$ & & IA \\
\hline & & $\bar{g}+k \sigma_{g}$ & $k$ & $P_{t}$ & $\beta$ & $1-P_{f}$ & $\beta$ & $1-P_{f}$ \\
\hline$g(L-W)$ & -0.00219 & 0.00005 & 1 & .8413 & 1.206 & .8862 & 1.199 & .8848 \\
\hline$g(L-W)$ & 0.00122 & 0.00031 & 2 & .9773 & 1.879 & .9699 & 1.859 & .9685 \\
\hline$g(L-W)$ & 0.000173 & -0.00075 & 3 & .9987 & 2.983 & .9986 & 2.939 & .9984 \\
\hline$g\left(C_{m}\right)$ & -0.000192 & -0.00003 & 1 & .8413 & 1.038 & .8504 & 1.050 & .8531 \\
\hline$g\left(C_{m}\right)$ & -0.00873 & -0.0050 & $2^{*}$ & .9773 & 3.471 & .9997 & 3.586 & .9998 \\
\hline$g\left(C_{m}\right)$ & -0.00695 & 0.00045 & 3 & .9987 & 4.164 & 1. & 4.165 & 1. \\
\hline
\end{tabular}

\section{Discussion of reliability assessment results}

The agreement between the robust design results and the reliability assessments degrades as the desired probability increases, which can be attributed to two factors. First, the term in the robust design constraint that determines the probability uses only information at the mean values of the random design variables to approximate a condition at the limit-state boundary. Second, although the random input variables are characterized by a normal distribution, the output functions are nonlinear; thus, there is no guarantee that the output functions are normally distributed. However, both the robust design and the reliability assessments are based on an assumption that the limit-state function is normally distributed. The extent to which these factors apply is also probably problem dependent.

\section{Timing Comparisons}

Since, for this problem, the computational time of the reliability assessment algorithms is negligible relative to that of the CFD and FEM analyses they require as input, the relative efficiency of the various reliability assessment methods can be made in terms of the number of calls to the function and derivative routines. That data is shown in Table 5 for several cases. The HL-RF RIA method and the PMA method require the same number of analyses for most of the cases. It is not surprising that the PMA-based RIA method requires more than the PMA since it is essentially a sequence of PMA analyses. From these results the case is not well made for use of the PRIA since it is consistently more expensive than the HL-RF method and the HL-RF did not exhibit any convergence difficulty.

\section{Sensitivity Derivatives from Reliability} Assessment

As a part of this reliability assessment, the sensitivity derivatives of the reliability information with respect to the mean values of the random design variables $\overline{b_{i}}$ are also obtained. These sensitivities are required for a gradient-based, reliability-based optimization procedure for which statements of the constraint appear as $\beta_{g}(\bar{b}) \geq \beta_{\text {target }}$ for the RIA approaches, or $g_{p}^{*} \leq 0$ for the PMA approach. The sensitivity derivatives of the reliability information $\beta$ and $g_{p}^{*}$ require no further differentiation than the first derivatives required for the reliability assessment. Their use as constraints does not require higher order derivatives as the Robust Optimization does. Sample reliability sensitivity derivative comparisons are shown in Tables 6 and 7 . The first column, labeled "DV", indicates the variable with respect to which the function is differentiated. The second and third columns identify the reliability assessment case by the constraint and desired reliability. The sensitivity results for the three reliability assessment methods are in the subsequent columns. The tables show the analytic sensitivity derivatives of the reliability index $\beta$, in the case of the two RIA methods, and the limit-state value $g_{p}^{*}$ in the case of the PMA. The tables also show the ratio of the analytic result to the derivative obtained by finite differences of two assessments for which the design point was perturbed slightly by the independent variable. Derivative results obtained by using the two RIA methods are found to agree very well. The 
Table 5 Timing comparisons of reliability assessment.

\begin{tabular}{cll|rr|rr|rr} 
& & & \multicolumn{2}{|c}{ HL-RF } & \multicolumn{2}{c}{ PMA } & \multicolumn{2}{c}{ PRIA } \\
nDV & Constraint & $k$ & Nfunc & Nderiv & Nfunc & Nderiv & Nfunc & Nderiv \\
\hline 2 & $g(L-W)$ & 1 & 3 & 3 & 3 & 3 & 6 & 5 \\
2 & $g(L-W)$ & 2 & 3 & 3 & 3 & 3 & 6 & 5 \\
2 & $g(L-W)$ & 3 & 3 & 3 & 3 & 3 & 6 & 5 \\
2 & $g(L-W)$ & 4 & 5 & 5 & 3 & 3 & 10 & 8 \\
2 & $g(V)$ & 3 & 3 & 3 & 3 & 3 & 9 & 7 \\
2 & $g(V)$ & 4 & & & 3 & 3 & 10 & 8 \\
4 & $g(L-W)$ & 1 & 3 & 3 & 3 & 3 & 6 & 5 \\
4 & $g(L-W)$ & 3 & 3 & 3 & 3 & 3 & 6 & 5 \\
4 & $g\left(C_{m}\right)$ & 1 & 4 & 4 & 3 & 3 & 6 & 5 \\
4 & $g\left(C_{m}\right)$ & 2 & 3 & 3 & 3 & 3 & 6 & 5 \\
4 & $g\left(C_{m}\right)$ & 3 & 3 & 3 & 3 & 3 & 9 & 7
\end{tabular}

agreement between the finite difference results and the analytic results is not very good for the $2 \mathrm{DV}$ cases shown in Table 6 . For the 4DV cases in Table 7 , however, the agreement is much better i.e., the ratio is closer to 1.000 . Some of the inaccuracy in the comparison can be attributed to the constraint tolerance specified in the RIA reliability assessment. The dagger symbols in the table indicate that a few of the finite difference values could not be obtained for comparison for the HL-RF RIA method because the RIA at the perturbed point failed to converge for at least one side of the central difference. These results indicate that the derivatives of the PMA and PRIA reliability assessment methods are suitable for use in a gradientbased design optimization process. The derivatives from HL-RF method are suitable when they are obtainable; but, the lack of assurance of convergence renders the method undesirable. Improvements to the HL-RF method has been suggested to correct the convergence problem; see, for example, reference 24.

\section{Concluding Remarks}

Assessment results from the three FORM-based reliability assessment methods were discussed and used to assess results from several cases of robust optimization of a $3-\mathrm{D}$ flexible wing. The three methods were qualitatively consistent for all the cases to which they were applied. Results from the two RIA methods were numerically very similar for all cases. The assessments showed good agreement with the target probabilities of the robust optimization when $k$ was less than about 3 . The analytical derivatives of the RIA methods were also nearly identical for all cases and consistent with the PMA method. The PMA and HL-RF methods required nearly the same computational time for all cases. The PMA-based RIA method was more computationally expensive; but, it showed no tendency to fail to converge as the HL-RF method did. The PMA and PRIA methods seem more suited for incorporation into a reliability-based design optimization (RBDO) process. The computational expense of RBDO rela- tive to robust design will likely be problem dependent. Robust optimization requires second-order sensitivity derivatives; hovever, RBDO requires inner loop optimizations to determine the MPP for active constraints.

\section{Acknowledgments}

The second author, G. J.-W. Hou, was supported in this work by NASA through several Tasks under contract NAS1-19858, NASA PO No. L-9291, and NASA PO No. L-11206 with the Old Dominion University Research Foundation.

\section{References}

${ }^{1}$ Zang, T., Hemsch, M. J., Hilburger, M. W., Kenny, S. P., Luckring, J. M., Maghami, P., Padula, S. L., and Stroud, W. J., "Needs and Opportunities for Uncertainty-Based Multidisciplinary Design Methods for Aerospace Vehicles," NASA TM 2002-211462, April 2002.

${ }^{2}$ Huyse, L., "Solving Problems of Optimization Under Uncertainty as Statistical Decision Problems," AIAA Paper 20011519, April 2001.

${ }^{3}$ Gumbert, C. R., Newman, P. A., and Hou, G. J.-W., "Effect of Random Geometric Uncertainty on the Computational Design of a 3-D Flexible Wing," AIAA Paper 2002-2806, July 2002 .

${ }^{4}$ Allen, M. and Maute, K., "Reliability-Based Design Optimization of Aeroelastic Structures," AIAA Paper 2002-5560, Sept. 2002

${ }^{5}$ Choi, K. K. and Youn, B. D., "On Probabilistic Approaches for Reliability-Based Design Optimization (RBDO)," AIAA Paper 2002-5472, Sept. 2002 .

${ }^{6}$ Haldar, A. and Mahadevan, S., Reliability Assessment Using Stochastic Finite Element Analysis, John Wiley \& Sons, Inc., New York, 2000.

${ }^{7}$ Hou, G. J.-W., "A Most Probable Point-Based Method for Reliability Analysis, Sensitivity Analysis, and Design Optimization," NASA CR in preparation, 2003.

${ }^{8}$ Bischof, C. H., Carle, A., Corliss, G. F., Griewank, A., and Hovland, P., "ADIFOR: Generating Derivative Codes from Fortran Programs," Scientific Programming, Vol. 1, No. 1, 1992, pp. 1-29.

${ }^{9}$ Bischof, C., Carle, A., Khademi, P., and Mauer, A., "Automatic Differentiation of FORTRAN," IEEE Computational Science \& Engineering, Vol. 3, No. 3, Fall 1996, pp. 18-32.

${ }^{10}$ Hasofer, A. M. and Lind, N. C., "Exact and Invariant Second-Moment Code Format," Journal of Engineering $\mathrm{Me}$ chanics Division ASCE, Vol. 100(EMI), 1974, pp. 111-121. 
Table 6 2DV reliability assessment sensitivity derivative comparisons.

\begin{tabular}{cll|rr|rr|rr} 
& & & \multicolumn{2}{|c|}{$\frac{\partial \beta}{\partial D V}, \mathrm{HL}-\mathrm{RF}$} & \multicolumn{2}{|c|}{$\frac{\partial \beta}{\partial D V}$, PRIA } & \multicolumn{2}{|c}{$\frac{\partial g^{*}}{\partial D V}$, PMA } \\
DV & Constraint & $k$ & analytic & ratio & analytic & ratio & analytic & ratio \\
\hline$c_{t}$ & $g(L-W)$ & 1 & 64.85 & 1.065 & 64.94 & & -1.152 & 1.091 \\
$c_{t}$ & $g(L-W)$ & 2 & 62.59 & 1.057 & 62.64 & 0.924 & -1.142 & 1.090 \\
$c_{t}$ & $g(L-W)$ & 3 & 67.28 & & 67.45 & 1.087 & -1.134 & 1.092 \\
$c_{t}$ & $g(L-W)$ & 4 & & & 76.22 & 0.997 & -1.115 & 1.073 \\
\hline$c_{t}$ & $g(V)$ & 3 & -77.19 & 1.060 & -77.25 & 1.013 & 1.058 & 1.001 \\
$c_{t}$ & $g(V)$ & 4 & -93.76 & & -93.93 & & 1.004 & 1.003 \\
$x_{t}$ & $g(L-W)$ & 1 & -35.38 & 0.926 & -35.34 & & &
\end{tabular}

Table 7 4DV reliability assessment sensitivity derivative comparisons.

\begin{tabular}{lll|rr|rr|rr} 
& & & \multicolumn{2}{|c|}{$\frac{\partial \beta}{\partial D V}$, HL-RF } & \multicolumn{2}{c|}{$\frac{\partial \beta}{\partial D V}$, PRIA } & \multicolumn{2}{c}{$\frac{\partial g^{*}}{\partial D V}$, PMA } \\
DV & Constraint & $k$ & analytic & ratio & analytic & ratio & analytic & ratio \\
\hline$\Gamma_{1}$ & $g(L-W)$ & 1 & -6.530 & 1.042 & -6.530 & 1.004 & .06952 & 0.992 \\
$\Gamma_{1}$ & $g(L-W)$ & 2 & -6.528 & 0.940 & -6.527 & 1.008 & .06917 & 0.998 \\
$\Gamma_{1}$ & $g(L-W)$ & 3 & -6.512 & 1.072 & -6.511 & 1.011 & .06881 & 0.998 \\
\hline$\Gamma_{1}$ & $g\left(C_{m}\right)$ & 1 & -9.457 & 0.942 & & & .05650 & 1.026 \\
$\Gamma_{1}$ & $g\left(C_{m}\right)$ & 2 & -9.778 & $\dagger$ & -9.778 & 1.000 & .05799 & 0.941 \\
$\Gamma_{1}$ & $g\left(C_{m}\right)$ & 3 & -9.984 & $\dagger$ & -9.984 & & .05949 & 1.036 \\
\hline$t_{r}$ & $g\left(C_{m}\right)$ & 3 & -471.5 & & -471.5 & & 2.802 & 1.008 \\
$z_{r}$ & $g\left(C_{m}\right)$ & 2 & -818.4 & $\dagger$ & -818.5 & 0.962 & 4.835 & 0.998 \\
$z_{r}$ & $g\left(C_{m}\right)$ & 3 & -816.3 & & -816.3 & 0.990 & 4.846 & 1.000 \\
$\Gamma_{2}$ & $g\left(C_{m}\right)$ & 2 & -22.47 & 1.099 & -22.47 & 0.958 & .1332 & 0.999 \\
$\Gamma_{2}$ & $g\left(C_{m}\right)$ & 3 & -23.02 & 1.018 & -23.02 & 1.003 & .1372 & 0.991 \\
\hline
\end{tabular}

$\dagger$ nonconvergent RIA for at least one side of the FD

${ }^{11}$ Rackwitz, R. and Fiessler, B., "Structural Reliability Under Combined Random Load Sequences," Computers and Structures, Vol. 9, No. 5, 1978, pp. 484-494.

${ }^{12}$ Barthelemy, J.-F. M. and Sobieszczanski-Sobieski, J., "Extrapolation of Optimum Design Based on Sensitivity Derivatives," AIAA Journal, Vol. 21, 1983, pp. 797-799.

${ }^{13}$ Barthelemy, J.-F. M. and Sobieszczanski-Sobieski, J., "Optimum Sensitivity Derivatives of Objective Functions in Nonlinear Programming," AIAA Journal, Vol. 21, 1983, pp. 913-915.

${ }^{14}$ Choi, K. K. and Youn, B. D., "Hybrid Analysis Method for Reliability-Based Design Optimization," Proceedings of the ASME 2001 Design Engineering Technical Conference and Computers and Information in Engineering Conference, Paper No. DETC2001/DAC-21044, Sept. 2001.

${ }^{15} \mathrm{Du}, \mathrm{X}$. and Chen, W., "A Most Probable Point-Based Method for Efficient Uncertainty Analysis," Design Manufacturing, Vol. 4, No. 1, 2001, pp. 47-66.

${ }^{16}$ Rumsey, C., Biedron, R., and Thomas, J., "CFL3D: Its History and Some Recent Applications," NASA TM-112861, May 1997.

${ }^{17}$ Sherman, L., Taylor, III, A., Green, L., Newman, P., Hou, G., and Korivi, M., "First- and Second-Order Aerodynamic Sensitivity Derivatives via Automatic Differentiation with Incremental Iterative Methods," Journal of Computational Physics, Vol. 129, No. 2, 1996, pp. 307-336.

${ }^{18}$ Taylor, III, A. C., Oloso, A., and Newman, III, J. C., "CFL3D.ADII (Version 2.0): An Efficient, Accurate, GeneralPurpose Code for Flow Shape-Sensitivity Analysis," AIAA Paper 97-2204, June 1997.

${ }^{19}$ Smith, R. E., Bloor, M. I. G., Wilson, M. J., and Thomas, A. T., "Rapid Airplane Parametric Input Design (RAPID)," Proceedings of the 12th AIAA Computational Fluid Dynamics Conference, San Diego, June 1995, pp. 452-462, also AIAA Paper $95-1687$.
${ }^{20}$ Jones, W. T. and Samareh-Abolhassani, J., "A Grid Generation System for Multidisciplinary Design Optimization," Proceedings of the 12th AIAA Computational Fluid Dynamics Conference, San Diego, June 1995, pp. 474-482, also AIAA Paper 95-1689.

${ }^{21}$ Bischof, C., Jones, W. T., Samareh-Abolhassani, J., and Mauer, A., "Experiences with the Application of the ADIC Automatic Differentiation Tool to the CSCMDO 3-D Volume Grid Generation Code," AIAA Paper 96-0716, Jan. 1996.

${ }^{22}$ Nguyen, D. T., "Finite Element Software for Multidisciplinary Design Optimization, Final Report," Tech. rep., Old Dominion University Research Foundation, Contract NAS1-19858, Task 69, NASA Langley Research Center, Hampton, VA, Nov. 1995.

${ }^{23}$ Hou, G., Arunkumar, S., and Tiwari, N. S., "First- and Second-Order Sensitivity Analysis of Finite Element Equations via Automatic Differentiation," Proceedings of the 7th AIAA/USAF/NASA/ISSMO Symposium on Multidisciplinary Analysis and Optimization, St. Louis, MO, Sept. 1998, pp. 454464, also AIAA Paper 98-4764.

${ }^{24}$ Zhang, Y. and der Kiureghian, A., "Two Improved Algorithms for Reliability Analysis," Proceedings of the IFIP WG7.5 working conference on reliability and optimization of structural systems, Chapman \& Hall, Weinheim, 1994, pp. 297-304. 